\title{
Effects of Delayed Treatment With Combined GDNF and Continuous Electrical Stimulation on Spiral Ganglion Cell Survival in Deafened Guinea Pigs
}

\author{
Verena Scheper, ${ }^{1 \star}$ Gerrit Paasche, ${ }^{1}$ Josef M. Miller, ${ }^{2}$ Athanasia Warnecke, ${ }^{1}$ \\ Nurdanat Berkingali, ${ }^{1}$ Thomas Lenarz, ${ }^{1}$ and Timo Stöver ${ }^{1}$ \\ ${ }^{1}$ Department of Otolaryngology, Medical University Hannover, Hannover, Germany \\ ${ }^{2}$ Kresge Hearing Research Institute, University of Michigan, Ann Arbor, Michigan
}

Electrical stimulation (ES) of spiral ganglion cells (SGC) via a cochlear implant is the standard treatment for profound sensor neural hearing loss. However, loss of hair cells as the morphological correlate of sensor neural hearing loss leads to deafferentation and death of SGC. Although immediate treatment with ES or glial cell line-derived neurotrophic factor (GDNF) can prevent degeneration of SGC, only few studies address the effectiveness of delayed treatment. We hypothesize that both interventions have a synergistic effect and that even delayed treatment would protect SGC. Therefore, an electrode connected to a pump was implanted into the left cochlea of guinea pigs 3 weeks after deafening. The contralateral untreated cochleae served as deafened intraindividual controls. Four groups were set up. Control animals received intracochlear infusion of artificial perilymph (AP/-). The experimental groups consisted of animals treated with $\mathrm{AP}$ in addition to continuous ES (AP/ES) or treated with GDNF alone (GDNF/-) or GDNF combined with continuous ES (GDNF/ES). Acoustically and electrically evoked auditory brain stem responses were recorded. All animals were killed 48 days after deafening; their cochleae were histologically evaluated. Survival of SGC increased significantly in the GDNF/- and AP/ES group compared with the AP/- group. A highly significant increase in SGC density was observed in the GDNF/ES group compared with the control group. Additionally, animals in the GDNF/ES group showed reduced EABR thresholds. Thus, delayed treatment with GDNF and ES can protect SGC from degeneration and may improve the benefits of cochlear implants. ๑ 2008 Wiley-Liss, Inc.

Key words: cochlear implant; glial cell line-derived neurotrophic factor; chronic electrical stimulation; delayed treatment

Damage to the sensory cells in the organ of Corti can be caused by various factors, such as noise, drugs, infections, mechanical injury, or aging. After the loss of the sensory epithelium, the downstream auditory neu- rons, the spiral ganglion cells (SGC), degenerate (Otte et al., 1978; Webster and Webster, 1981; Spoendlin, 1984; Nadol and Hsu, 1991).

The functional state and the number of surviving SGC are among the factors that determine the success of the cochlear implant treatment (Gantz et al., 1993). Deprivation of both excitatory activity and neurotrophic factors (NTF) have been identified as etiological factors for the loss of auditory neurons after deafness (Shepherd et al., 2005). It has been shown that excitation of the auditory nerve by electrical stimulation (ES) greatly reduces the hair cell loss-related degeneration of SGC (Lousteau, 1987; Hartshorn et al., 1991; Leake et al., 1991; Mitchell et al., 1997). However, this neuroprotective effect of ES has been objected by other authors (Araki et al., 1998; Shepherd et al., 1994; Li et al., 1999). NTFs, such as neurotrophin-3, glial cell linederived neurotrophic factor (GDNF), brain-derived neurotrophic factor (BDNF), and ciliary-derived neurotrophic factor, can reduce deafferentation-induced SGC degeneration (Ernfors et al., 1996; Staecker et al., 1996; Miller et al., 1997; Ylikoski et al., 1998; Kuang et al., 1999; Yagi et al., 2000; Shinohara et al., 2002; Nakaizumi et al., 2004). Simultaneous in vivo treatment of SGC with ES and BDNF (Shepherd et al., 2005) or GDNF transgene delivery from an adenoviral vector (Kanzaki et al., 2002) resulted in a significant enhancement of trophic effects. In these studies, NTF and ES were delivered shortly after deafening, before the onset of SGC degeneration.

Contract grant sponsor: European Union, BIOEAR-project; Contract grant number: QLRT-2001-01563.

^Correspondence to: Verena Scheper, Department of Otolaryngology, Medical University Hannover, Carl-Neuberg-Str. 1, 30625 Hannover, Germany. E-mail: Scheper.Verena@MH-Hannover.de

Received 21 April 2008; Revised 10 October 2008; Accepted 13 October 2008

Published online 15 December 2008 in Wiley InterScience (www. interscience.wiley.com). DOI: 10.1002/jnr.21964 
The success that can be achieved by the use of cochlear implants is related to the duration of deafness (Tyler and Summerfield, 1996). After the loss of the sensory epithelium, SGC degeneration obviously occurs in two phases with different underlying biomolecular mechanisms. For example, drug-deafened guinea pigs show a significant degeneration of SGC over the first 2 weeks after hair cell loss (Jyung et al., 1989). A further significant degeneration occurs over the ensuing 2 months, leading to a stable neuronal population (approximately $13 \%$ of the SGC population of healthy animals; Webster and Webster, 1981; Jyung et al., 1989). Delayed treatment of experimentally deafened guinea pigs with BDNF and ciliary-derived neurotrophic factor axokine-1 (Yamagata et al., 2004) or BDNF and fibroblast growth factor 1 (Miller et al., 2007) beginning after degenerative and apoptotic processes have started increases the survival of SGC. Even delayed ES of the auditory nerve of kittens deafened immediately after birth led to a significant protection of SGC (Leake et al., 1999). It was also shown that delayed ES could rescue SGC (Miller and Altschuler, 1995); however, a higher level of ES was required when compared with the level of ES that is effective immediately after deafness.

To better model the human condition in which considerable degeneration of the SGC population has often already occurred before implantation, the effectiveness of a simultaneous treatment that uses both interventions-ES and GDNF-delayed needs to be assessed. Therefore, we examined for the first time the combined effects of delayed intracochlear application of GDNF $(100 \mathrm{ng} / \mathrm{ml})$ and $\mathrm{ES}$ on the survival of SGC in systemically deafened guinea pigs. The novel aspect of this study is that experimental treatments were delayed by 3 weeks after deafening, when about $15 \%$ of the SGC population (Webster and Webster, 1981; Jyung et al., 1989) are expected to have already degenerated.

\section{MATERIALS AND METHODS}

\section{Experimental Subjects}

The study was conducted in accordance with the German "Law on Protecting Animals" and with the European Communities Council Directive 86/609/EEC for the protection of animals used for experimental purposes. All experiments were approved by the Institutional Animal Care and Research Advisory Committee at the Medical University Hannover and were permitted by the local government (LAVES, registration 02/558 and 07/1389).

Thirty male pigmented guinea pigs (Charles River WIGA GmbH, Sulzfeld, Germany), weighing between 250 and $450 \mathrm{~g}$, were used in this study. Inclusion criteria were normal hearing thresholds ( $\leq 50 \mathrm{~dB}$ SPL) assessed by acoustically evoked auditory brain stem response (AABR) and a threshold shift of $60 \mathrm{~dB}$ or more after the deafening procedure. All procedures were performed under general anesthesia with xylazine (10 $\mathrm{mg} / \mathrm{kg}$, i.m.) and ketamine $(40 \mathrm{mg} / \mathrm{kg}$, i.m.). Local prilocaine anesthesia was used for the comfort of the animals.
Normal hearing animals $(n=6)$ were taken for assessment of SGC perikaryal diameter in nontreated cochleae. The remaining animals $(n=24)$ were deafened and after 3 weeks divided into four treatment groups. Animals receiving artificial perilymph (AP) alone (AP/- group; $n=7$ ) served as controls. Animals of the AP/ES group $(n=6)$ received AP in combination with continuous ES. Animals of the GDNF/group were administered the NTF GDNF $(n=6)$ alone and of the GDNF/ES group in combination with ES $(n=5)$.

\section{Deafening Procedure}

Animals were chemically deafened on day 0, immediately after the first AABR recording. Single coadministration of the ototoxic agents kanamycin $(400 \mathrm{mg} / \mathrm{kg}$, s.c.) and ethacrynic acid (40 mg/kg, i.v.) was used for the systemic deafening. The ethacrynic acid infusion was delayed by $2 \mathrm{hr}$ after kanamycin injection, according to the method described by West et al. (1973). This procedure resulted in complete sensory cell loss and, as a consequence, induction of degeneration processes of the SGC and their nerve fibers. The pattern of SGC degeneration and the density of SGC in the normal guinea pig inner ear have previously been described in detail (Webster and Webster, 1981; Jyung et al., 1989). Deafness was confirmed on day $5 / 6$ as well as on day 21 (just before implantation) by AABR.

\section{AABR}

Hearing thresholds were determined and recorded by AABR with the Nicolet Viking IV system (Nicolet Biomedical Inc., Madison, WI). Computer-generated alternating polarity voltage pulses ("click" stimuli) of $100 \mu \mathrm{sec}$ duration and $10.0 \mathrm{~Hz}$ repetition rate were delivered monaurally through a transducer and eartips (polyurethane foam eartips, $13 \mathrm{~mm}$, TIP-300 Tubal Insert Phone, Nicolet Biomedical Inc.) positioned in the external ear canal. To record the neurological responses, subdermal needle electrodes $(1.0 \mathrm{M}$, Nicolet Biomedical) were placed at the vertex (common positive), right and left mastoid (reference), and in the right lower hind limb (ground). Click stimuli from $90 \mathrm{~dB}$ SPL decreasing in $10 \mathrm{~dB}$ steps ( $5 \mathrm{~dB}$ steps to bracket threshold) were applied. The contralateral ear was masked with white noise $30 \mathrm{~dB}$ below stimulus level. The responses were filtered $(0.1-3 \mathrm{kHz})$, amplified, and averaged. Threshold was defined as the lowest click stimulus required to evoke a visually replicable waveform. AABRs were assessed bilaterally on day 0 (before the deafening procedure), on day 5/6, and on day 21 to confirm the success of the deafening procedure.

\section{Surgical Implantation Procedure of the Electrode and the Pump}

The electrode-cannula combination (MedEl GmbH, Innsbruck, Austria) consisted of an active electrode, a reference electrode, a percutaneous connector, and a silicone tube (Fig. 1). The active electrode was a Teflon-insulated platinum-iridium wire with $50 \mu \mathrm{m}$ diameter connected to a single ball contact $(250 \mu \mathrm{m}$ in diameter). The system was cast in silicone that also contained a drug-delivery canal $(150 \mu \mathrm{m}$ diameter). The reference electrode consisted of a Teflon-insulated 


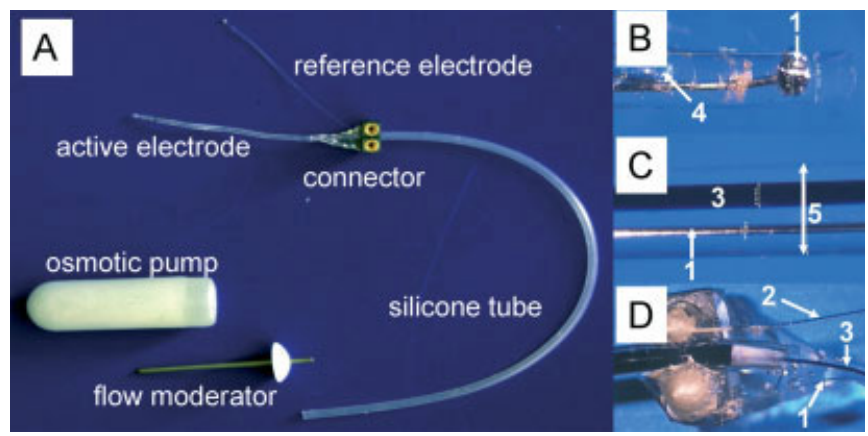

Fig. 1. Illustration of the electrode-cannula device. A: Electrode micropump system, pump (Alzet osmotic pumps) and the flow moderator that connects the two components. B: Tip of the active electrode. C: Center piece of the active electrode and application canal embedded in silicone. D: Ventral view of connector. 1: Active electrode. 2: Reference electrode. 3: Drug channel. 4: Channel aperture. 5: Silicone. [Color figure can be viewed in the online issue, which is available at www.interscience.wiley.com.]

$150 \mu \mathrm{m}$ diameter platinum-iridium wire. A mini osmotic pump (Alzet model 2002; Durect Corp.; infusion rate $0.5 \mu \mathrm{l}$ / $\mathrm{hr}$, suitable for a 14-day delivery) was connected to the silicone tube and was used for local fluid delivery into the cochlea. In the $\mathrm{AP} /-$ group and the $\mathrm{AP} / \mathrm{ES}$ group, the pumps were filled with AP $(145 \mathrm{mM} \mathrm{NaCl}, 2.7 \mathrm{mM} \mathrm{KCl}, 2.0 \mathrm{mM}$ $\mathrm{MgSO}_{4}, 1.2 \mathrm{mM} \mathrm{CaCl}$, $5.0 \mathrm{mM}$ HEPES, $0.1 \%$ guinea pig serum albumin). GDNF (Amgen) primed pumps were used for the GDNF/ - and the GDNF/ES group (100 ng GDNF dissolved in $1 \mathrm{ml} \mathrm{AP})$. All osmotic pumps were exchanged after 13 days (experimental day 34) to continue infusion of GDNF or AP until experimental day 48.

All deafened guinea pigs were implanted unilaterally (left ear) with the electrode-cannula device (Fig. 1) connected to the mini osmotic pump for direct ES of the auditory nerve and drug delivery into the scala tympani. According to Mitchell et al. (1997), epidural recording electrodes and restraint bolt holding screws were implanted. A subcutaneous pocket between the scapulae was formed to accommodate the pump. The middle ear was exposed, and the round window membrane was incised (Stover et al., 1999). The primed pump was attached to the fluid-filled cannula and positioned in the subcutaneous pocket. All screws, the tube, and the percutaneous connector were secured on the top of the skull with methyl methacrylate (Meta Fast Resin, J. Morita Europe GmbH, Dietzenbach, Germany). The active electrode was inserted through the round window approximately $3 \mathrm{~mm}$ into the scala tympani. The reference electrode was placed at the bony wall of the bulla. Before the sealing of the bulla defect, EABR were recorded to confirm functionality of the electrode array. Both electrodes were fixed when the bony bulla defect was closed with carboxylate cement (Durelon, ESPE Dental AG, Seefeld, Germany). The wound was closed in two layers.

\section{Electrically Evoked Auditory Brain Stem Response (EABR)}

EABR were recorded directly after electrode implantation to determine the electrical hearing thresholds, and then weekly for 4 weeks on experimental days 21, 28, 34, 41, and 48. Computer-generated monophasic current pulses (Vicking IV, Nicolet Biomedical Corp., Madison, WI) were presented through a 10-MHz pulse generator (TGP $11010 \mathrm{MHz}$ Pulse Generator, Thurlby Thandar Instruments, Huntingdon, UK). With a custom-made modifier, the stimuli were converted to alternating monophasic pulses. Responses were recorded between the epidural electrodes at the vertex $(1 \mathrm{~cm}$ posterior to bregma), midline (2 $\mathrm{cm}$ anterior to bregma), and, as a ground, $1 \mathrm{~cm}$ lateral to bregma, ipsilateral to the implant (Mitchell et al., 1997).

The averaged response to 500 presentations of a given stimulus was recorded. The stimulus level was adjusted in steps of $10 \mu \mathrm{A}$. Threshold was defined as the lowest stimulus level that evoked a $1 \mu \mathrm{V}$ or greater replicable waveform.

\section{Chronic ES}

Beginning on day 24 (3 days after implantation), animals in the GDNF/ES and AP/ES groups received continuous pulsatile ES for 24 days. Biphasic charge balanced pulses, 100 $\mu$ sec per phase, $250 \mathrm{~Hz}$ at a $40 \%$ duty cycle, were presented 8 $\mathrm{dB}$ above the electrical response threshold. The battery-powered, wearable stimulators were provided by the University of Michigan, Ann Arbor, according to Mitchell et al. (1997) and Kanzaki et al. (2002).

\section{Histological Procedures}

Immediately after EABR recording on day 48, animals were killed by transcardial perfusion with $200 \mathrm{ml}$ of phosphate-buffered saline, followed by $200 \mathrm{ml}$ of $4 \%$ glutardialdehyde in phosphate-buffered saline. The temporal bones were removed and the middle ears were examined for infections, tissue reaction, and electrode placement. A hole was cautiously drilled in the apex of the cochlea, and an intrascalar perfusion through the round window with glutardialdehyde was performed. Cochleae were than placed in the fixative for $24 \mathrm{hr}$ at $4^{\circ} \mathrm{C}$. After rinsing with $20 \%$ trisodium citrate for 1 $\mathrm{hr}$, the cochleae were decalcified in a trisodium citrate-formic acid mixture for 14 days; the solution was changed every day. Cochleae were than dehydrated through a graded series of alcohols (70-100\% ethanol). After embedding in paraffin, the cochleae were serially sectioned at $5 \mu \mathrm{m}$ in a midmodiolar plane. Every section was mounted on a glass slide and stained with hematoxylin and eosin. Midmodiolar sections were used for the quantitative analysis of SGC density because they contain six to seven profiles of Rosenthal's canal.

We specified the cochlear turns from base to apex as follows: lower and upper basal turn $(\mathrm{lb}, \mathrm{ub})$, first middle turn (1.m), second middle turn (2.m), third middle turn (3.m), fourth middle turn (4.m), and apical turn (a) (Fig. 2). Systematic random sampling was used: the first midmodiolar section was randomly selected and then every fifth proximate section was chosen; thus, five most midmodiolar sections were analyzed, each separated by $25 \mu \mathrm{m}$. The number of SGC was assessed for each of the six or seven cross-sectional profiles of Rosenthal's canal on each of the five sections for each cochlea. Perikaryal diameter were assessed from five randomly selected SGC (if available) per Rosenthal's canal cross section. 




Fig. 2. Midmodiolar cochlear cross section $(5 \mu \mathrm{m})$ showing the different locations at which SGC were examined. The SGC density was calculated for the profiles of Rosenthal's canal (RC) belonging to the lower basal turn (lb), upper basal turn (ub), first, second, third, and fourth middle turn (1.m, 2.m, 3.m, 4.m), and the apical turn (a). The hair cell loss 48 days after deafening is apparent (arrows). The scala tympani of the lower basal turn is nearly completely filled with connective tissue (ct). The hollow space indicates where the electrode (e) was placed. Magnification, $15 \times$. [Color figure can be viewed in the online issue, which is available at www.interscience.wiley.com.]

The perikaryal diameter was measured nearly parallel to the osseus spiral lamina in the longitudinal axis of the neurons. This axis was easy to discern because the SGC were more oval rather than round (Fig. 4). The measurement and the quantification were performed microscopically at a magnification of $200 \times$ (Olympus CKX41, Hamburg, Germany). Images were taken with a CCD camera (Colorview XS, SIS, Muenster, Germany) and processed by an image analysis program (analySIS Version. 3.2, SIS). Only SGC with a minimum perikaryal diameter of $12 \mu \mathrm{m}$ and a discernible nucleus were counted and included for analysis. The cross-sectional area of each profile of Rosenthal's canal was determined and was used to calculate the SGC density (cells/10,000 $\mu^{2}{ }^{2}$ ). Both cross-sectional areas of each profile of Rosenthal's canal and SGC counts were performed in a blinded manner (C.R. and P.E.; see acknowledgment). Because SGC counting and area measurements were not always reliable at the most apical sites, these measurements were combined with the fourth middle turn if available.

\section{Statistical Analysis}

All data passed the normality test. The nonparametric Wilcoxon signed ranks test was applied for analysis of the SGC density differences between the treated left side and the untreated right side within the animals of one group. To assess significant differences among the different experimental groups, Bonferroni's multiple comparison test was used.

\section{RESULTS}

\section{AABR and Deafening}

Normal hearing animals typically demonstrated an AABR threshold of $35 \mathrm{~dB}$ SPL. The application of ototoxic agents (kanamycin and ethacrynic acid) resulted in an AABR threshold shift in all animals of at least $60 \mathrm{~dB}$ or more. The success of the deafening procedure is also reflected histologically in a nearly complete loss of both inner and outer hair cells. Figure 2 illustrates the elimination of the organ of Corti observed in each of the treatment groups. The left and the right cochleae of all cohorts showed destroyed hair cells over a distance of more than two turns, from the lower basal turn to the third middle turn. Apical to this level, the damage to the organ of Corti was variable. In the right deafened and untreated cochleae of all groups, the mean SGC density ranged from 2.84 to $3.03 \mathrm{SGC} / 10,000 \mu^{2}$ (Table I) after 48 days of deafness.

\section{EABR Thresholds}

EABRs were recorded on day 21 and weekly throughout the experiment to monitor threshold changes related to the treatment. Representative EABR recordings are shown in Figure 3A,B, evoked from a guinea pig of the GDNF/ES-treated group by various intensities of ES. Figure 3A illustrates the EABR immediately after implantation (day 21), and Figure 3B demonstrates the response on day 48, showing a decrease of thresholds after treatment with GDNF and ES. Figure 3C depicts the EABR threshold changes over the 24-day stimulation period for both electrically stimulated groups (AP/ES, GDNF/ES). All animals treated with GDNF in combination with ES exhibited a decrease of thresholds over the whole study period (48 days) whereas animals from the AP/ES group initially showed an increase of thresholds until day 34, and thereafter a slight decrease. This resulted in an overall increase of EABR thresholds in the $\mathrm{AP} / \mathrm{ES}$ group (Fig. 3D).

\section{Neuronal Survival}

Representative histological sections from all four treatment groups are shown in Figure 4. The SGC survival differed between the treated and untreated control cochleae within one group and between the four experimental groups.

Comparison of the left (treated) side with the right (untreated) side. Spiral ganglion cells (SGCs) were counted in the sections from cochleae of the untreated right ears and from the treated left ears. SGC numbers (mean \pm standard deviation [SD]) were com- 
TABLE I. Density and Survival in Treated Cochleae Compared With Associated Deafened Side ${ }^{\dagger}$

\begin{tabular}{|c|c|c|c|c|c|c|}
\hline Group & \multicolumn{2}{|c|}{ Left, treated side } & \multicolumn{2}{|c|}{ Right, control side } & $\begin{array}{c}\text { Difference } \\
\left(\text { cells } / 10,000 \mu \mathrm{m}^{2}\right)\end{array}$ & $P$ value \\
\hline $\mathrm{AP} / \mathrm{ES}$ & 3.60 & 125.87 & 2.86 & 100 & 0.74 & $0.0270^{\star}$ \\
\hline GDNF/- & 4.09 & 136.21 & 3.01 & 100 & 1.08 & $0.0089^{\star}$ \\
\hline GDNF/ES & 5.49 & 181.18 & 3.03 & 100 & 2.46 & $0.0010^{\star}$ \\
\hline
\end{tabular}

${ }^{\dagger}$ Mean spiral ganglion cell (SGC) density and mean SGC survival (experimental ear/control ear $\times 100$ ) are shown. The SGC density of the control side was considered $100 \%$ survival and used for the comparison of SGC survival of the treated side.

$\star P<0.05$.

$\star \star$ Not significant.

pared between the left and the right side (Table I). The results showed that delayed administration of GDNF alone or in combination with ES led to a significant increase of the mean SGC density compared with the contralateral untreated right cochleae $(4.09$ vs. 3.01 $\mathrm{SGC} / 10,000 \mu \mathrm{m}^{2}$ and 5.49 vs. $3.03 \mathrm{SGC} / 10,000 \mu \mathrm{m}^{2}$, respectively; $P<0.01$, Table I). Also, combined treatment with AP and ES caused a significant increase in SGC density compared with the deafened right control cochleae (3.60 vs. $2.86 \mathrm{SGC} / 10,000 \mu^{2}, P<0.05$ ). However, animals receiving AP only showed a decrease in SGC density of the implanted left side compared with the untreated right side (approximately $-16 \%$ ).

Comparison of treatment groups. The density of protected SGC is the difference between the density of surviving SGC on the treated (dSGCi) left side and the untreated (dSGCni) right side (dSGCi - dSGCni). We compared the mean density of protected SGC of all four treatment groups (Fig. 5). ES (AP/ES) enhanced the survival of SGC when compared with treatment with AP alone (0.74 vs. -0.45 protected SGC/10,000 $\left.\mu^{2} ; P<0.05\right)$. Infusion of GDNF was even more potent in increasing the number of surviving SGC (1.08 protected SGC in $\left.10,000 \mu \mathrm{m}^{2} ; P<0.01\right)$ and appeared to provide greater SGC protection than treatment with $\mathrm{ES}$ alone (1.08 vs. $\left.0.74 \mathrm{SGC} / 10,000 \mu^{2}\right)$. However, the greatest degree of SGC protection was seen after combined treatment with GDNF and ES $(2.46 \pm 1.15$ / $10,000 \mu^{2} ; P<0.001 ;$ Fig. 5; Table I). The protection of SGC achieved by the combined treatment with GDNF and ES was statistically significant when compared with the success achieved when either of the two factors was used alone $(P<0.01$ vs. ES; $P<0.05$ vs. GDNF).

When subtracting the mean density of protected $\mathrm{SGC}$ of the AP/ - group from the mean density of protected SGC of the other groups (AP/ES group: 1.19 $\mathrm{SGC} / 10,000 \mu \mathrm{m}^{2}$; GDNF/ - group: $1.53 \mathrm{SGC} / 10,000$ $\mu^{2}$; GDNF/ES group: $2.91 \mathrm{SGC} / 10,000 \mu \mathrm{m}^{2}$ ), it is evident that the combined effect after coadministration of GDNF and ES is greater than the sum of effects of the two individual factors (1.19 (AP/ES group) +1.53 $(\mathrm{GDNF} /-$ group $)=2.72)$. This suggests an interactive synergistic effect of these two survival factors when applied simultaneously.
Basal turn vs. apical turn. Comparison of the SGC survival in the basal turn (lower and upper basal turn + first middle turn) vs. the middle and apical turn (second + third + fourth middle turn + apical turn) of the cochleae of all treatment groups showed a clear difference in SGC survival (Fig. 6). In the GDNF/ES group, the GDNF/ - group and the AP/ES group the mean density of protected SGC in the basal turn was higher than in the associated middle and apical turns. This difference is significant in the GDNF/ES cohort $(P<0.05)$. By contrast, the control cochleae $(\mathrm{AP} /-)$ showed a higher SGC density in the middle and apical turns than in the basal turn.

\section{Perikaryal Diameter}

Figure 7 depicts the SGC perikaryal diameter for each experimental group of the study and for normal hearing guinea pigs. The perikaryal diameter in the AP/ - group was $17.27 \pm 1.12 \mu \mathrm{m}$. The AP/ES-treated cochleae exhibit a mean perikaryal diameter of $17.79 \pm$ $1.05 \mu \mathrm{m}$, whereas SGC in the GDNF/--treated cochleae were $17.23 \pm 0.80 \mu \mathrm{m}$, and cells in the GDNF/ES cochleae averaged $17.39 \mu \mathrm{m} \pm 1.00 \mu \mathrm{m}$ SD. No significant differences in soma diameter were observed across the treatment groups. Perikaryal diameter of normal hearing animals were $15.20 \pm 0.23 \mu \mathrm{m}$ and differed statistically significant from the data of the AP/ $-(P<$ $0.01)$, AP/ES $(P<0.001)$, GDNF $(P<0.05)$, and GDNF/ES $(P<0.01)$ groups.

\section{Connective Tissue Growth}

Connective tissue growth around the electrode was observed in 17 of 24 implanted cochleae. The tissue formation was variable, ranging from no tissue growth to total filling of the scala tympani (Fig. 2). The tissue reached in some cases from the lower basal turn up to the third middle turn. In contrast to Shepherd et al. (2005), who detected fine fibrous tissue in cochleae treated chronically with BDNF- but not in AP-treated animals, we observed no differences in the growth of connective tissue between the groups. 
A
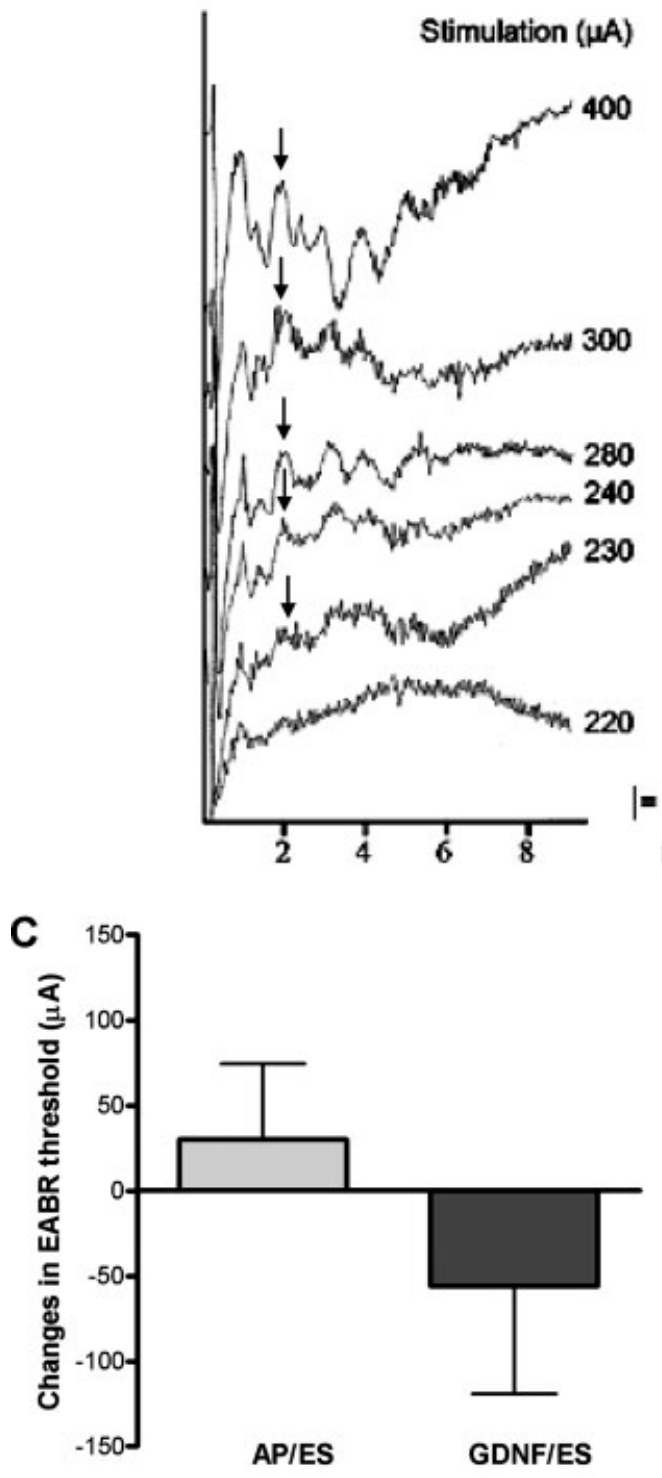

Fig. 3. A,B: Averaged electrophysiological wave forms elicited by varied intensities of ES from an animal of the GDNF/ES group. Display: $1 \mu \mathrm{V}$; A: Recordings were made 21 days after deafening. Thresholds were defined on the basis of P III responses (black arrows). The threshold for this subject was defined as $230 \mu \mathrm{A}$. B: EABR response of the same animal on day 48. The threshold decreased to $140 \mu \mathrm{A}$. C: Mean $\pm \mathrm{SD}$ changes in EABR thresholds

\section{DISCUSSION}

Typically, patients receive their cochlear implant at least 6 month after deafening. It has been shown in animals that within this period a significant loss of SGC has already occurred (Jyung et al., 1989). Although the degeneration of SGC in humans is assumed to be much slower (Otte et al., 1978), a significant loss of SGC may have occurred by the time of cochlear implantation. The
B
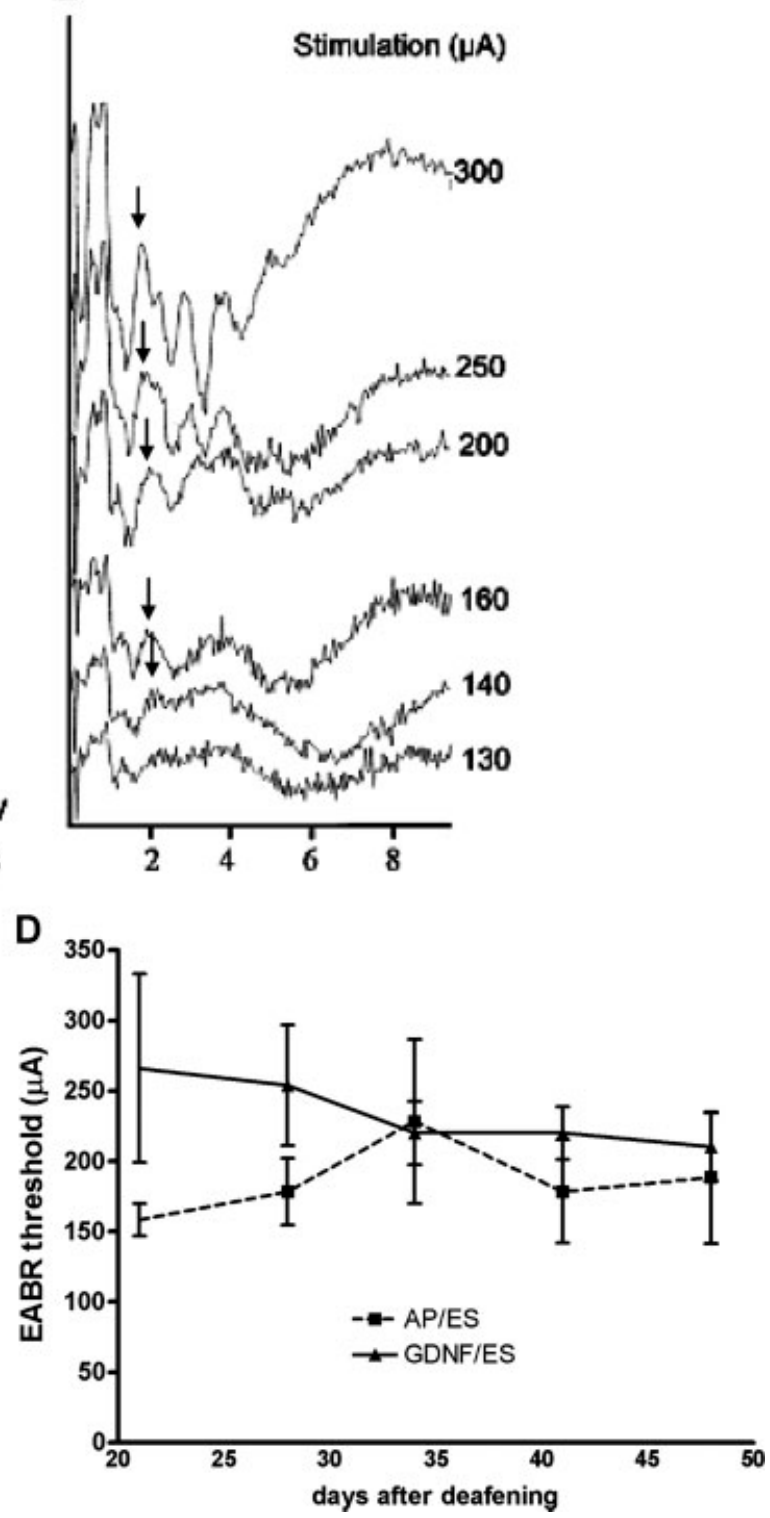

from day 21 to day 48 from animals treated with delayed AP/ES or GDNF/ES. EABR threshold before ES (d21) is displayed as baseline. There are no statistical differences between the two groups. However, the combined GDNF/ES treatment showed a tendency toward a continuous decrease in EABR thresholds throughout the study. By contrast, EABR thresholds displayed an increase in AP/ES-treated animals over time (D).

benefits that can be achieved by the use of cochlear implants are, among other factors, dependent on the survival and the excitability of SGC. Immediate intervention with NTFs and ES may result in enhanced survival and sensitivity of deafferented neural tissue in vivo. However, it is of major importance to investigate whether delayed interventions have a similar efficacy. Recently, it was demonstrated that delayed ES and 
Fig. 4. Representative midmodiolar cross sections of Rosenthal's canal of the first middle turn of left cochleae from all four treatment groups. Arrows indicate SGC. A: Control group (deafened and AP treated) showing the complete loss of healthy SGC. Only degenerated SGC are discernible. B: Section from an AP/ES-treated animal with better sustainment and density of SGC. C: GDNF treatment also led to a higher SGC density. D: Compared with the other groups of study, the GDNF/ES-treated group showed the highest SGC density. When the cell morphology evident in all four micrographs is compared, no visible differences in size or degree of shrinkage can be found. [Color figure can be viewed in the online issue, which is available at www.interscience.wiley.com.]
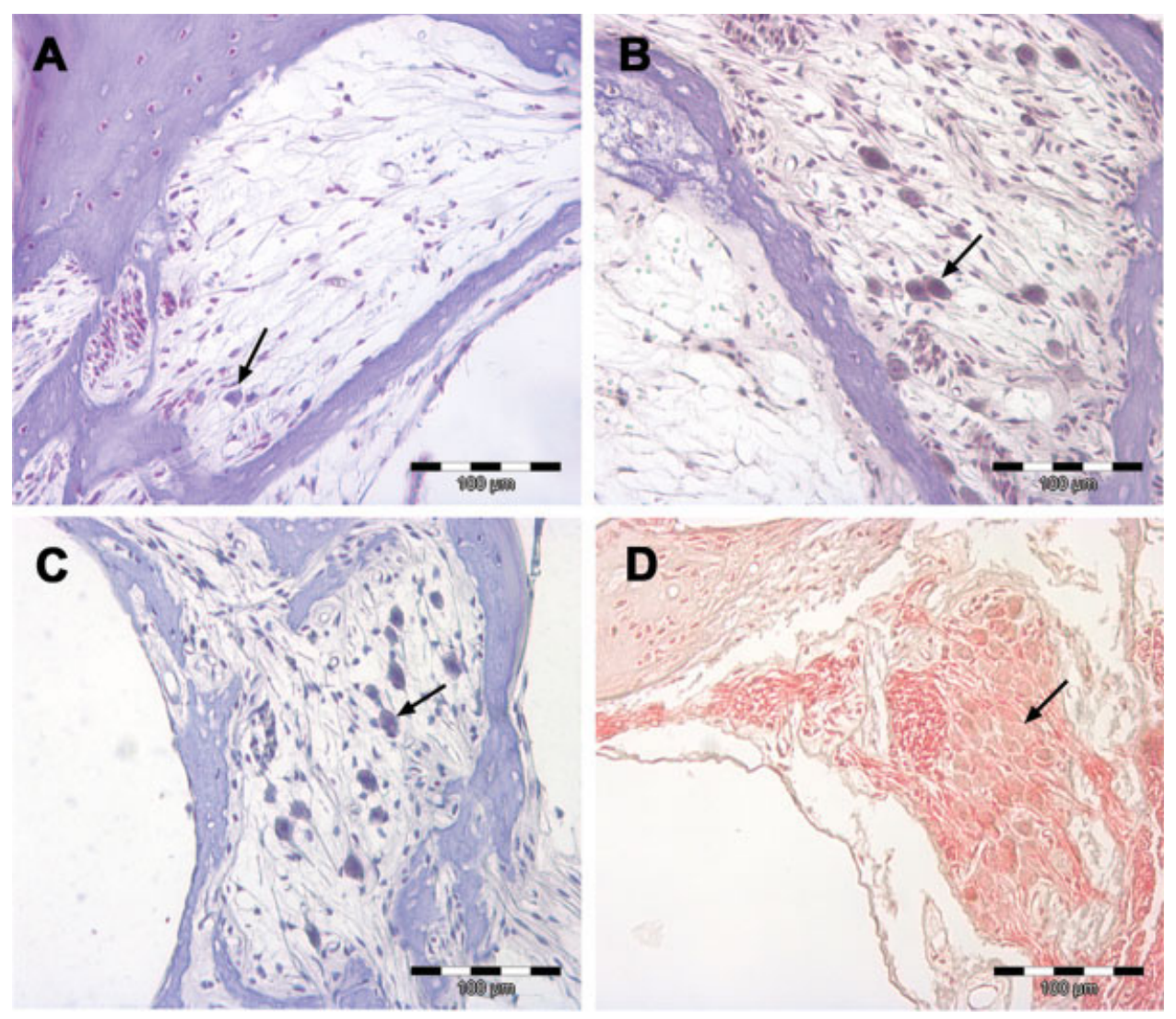

BDNF application lead to functional and neuroanatomical responses in deafened rats (Song et al., 2008).

The present work examined the functional and anatomical changes induced by a delayed treatment with GDNF (100 ng/ml) and/or ES in deafened guinea pig cochleae. Our results confirm previous studies demonstrating that GDNF and ES alone can increase SGC survival in deafened guinea pigs. Additionally, they also provide evidence of a more than additive effect after combined treatment with GDNF infusion and chronic ES. We demonstrated for the first time that this significant effect of GDNF in combination with ES can be observed even after a treatment delay of 21 days $(P<$ 0.01 , Fig. 5), when significant degeneration of SGC has already occurred.

\section{SGC Survival in the GDNF Group}

According to the suggestions of Jyung et al. (1989) and Webster and Webster (1981), our treatment delay exceeded 14 days to enable the development of significant SGC degeneration. This increased delay between deafening and initiation of treatment was chosen in order to verify that protection of SGC can also be induced with treatment interventions starting once degeneration has already progressed.

Webster and Webster (1981) reported that a combination of kanamycin and ethacrynic acid caused a significant loss of SGC in guinea pigs after 2 weeks. Vari- ous studies demonstrated that GDNF application to the inner ear has a protective effect on SGC after deafening. Intracochlear GDNF infusion in noise exposed guinea pigs lead to a significantly enhanced SGC survival when treatment was started 4 days after the deafening procedure (Ylikoski et al., 1998). Marujama and colleagues treated guinea pigs with GDNF 2 days after chemical deafening and observed a neuroprotective effect (Maruyama et al., 2007). Furthermore, adenoviral vectormediated GDNF application 4-7 days after ototoxic treatment has the ability to rescue SGC from degeneration (Yagi et al., 2000; Kanzaki et al., 2002). Our study corroborates these results and proves the effectiveness of GDNF even after a delayed intervention.

\section{SGC Survival in the ES/AP Group}

Miller and Altschuler (1995) showed that ES (200 $\mu \mathrm{A})$ starting 2 weeks after deafness is effective for SGC protection. We found a significantly increased density of SGC in the electrically stimulated left cochleae when compared with the associated untreated right cochleae $(P$ $<0.05$, Table I). Furthermore, the density of protected SGC in animals treated with AP/ES differed significantly $(P<0.05)$ from the density of SGC determined in control animals treated only with AP. Thus, continuous ES, delayed by 24 days, still protects SGC from degeneration after hair cell loss. 


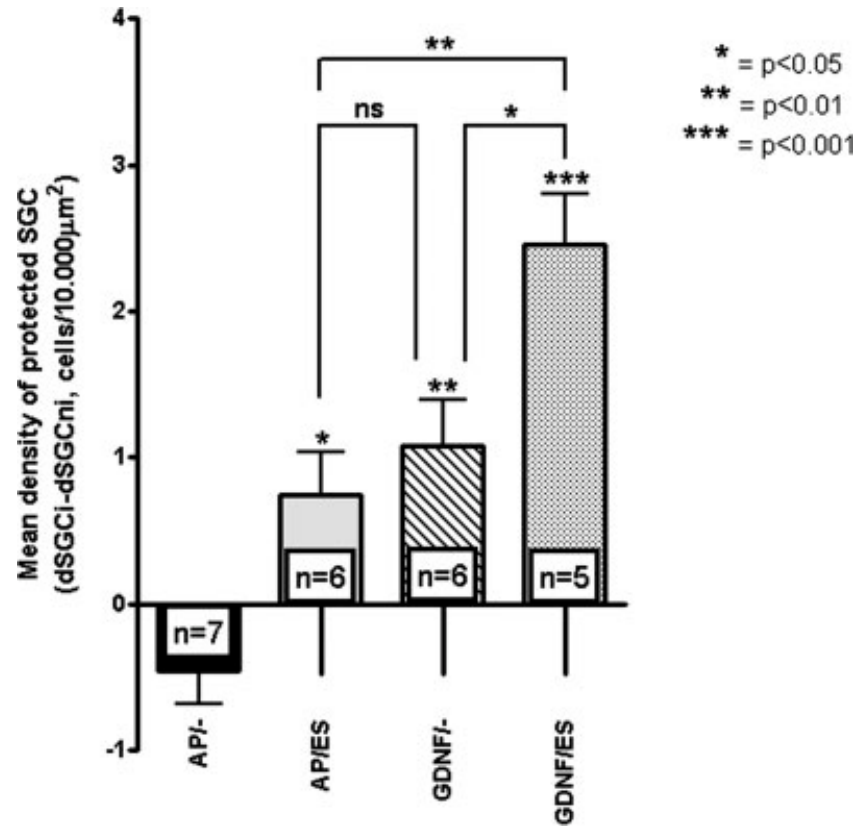

Fig. 5. Mean density of protected SGC in the four experimental groups. The vertical bars indicate standard deviation. SGC density was significantly higher in the AP/ES $(P<0.05)$, GDNF/ - $(P<$ $0.01)$, and GDNF/ES group $(P<0.001)$ when compared with the control group $(\mathrm{AP} /-)$. The GDNF treatment led to a slight but not significant increase of SGC survival when compared with ES treatment alone. GDNF/ES led to a more than tripled density of protected SGC when compared with treatment with ES alone $(P<$ 0.01 ), whereas the GDNF/ES treatment was approximately twice as effective compared with GDNF alone $(P<0.05)$.

The earlier the treatment is started after deafening, the higher the survival of SGC that can be achieved. For example, already at 2 days after ototoxic drug treatment, Lousteau (1987) and Hartshorn et al. (1991) stimulated guinea pigs for $1 \mathrm{hr} /$ day for 45 days and for $2 \mathrm{hr} /$ day for 9 weeks, respectively. In both groups, a $50 \%$ or higher increase in SGC density in the electrically stimulated cochleae was determined when compared with the deafened untreated side. In contrast, we observed a $25 \%$ higher SGC density in stimulated cochleae. The treatment delay is the factor that reduces the number of SGC, and this is consistent with Mitchell et al. (1997), who started ES of guinea pigs 8 days after deafening. They achieved a mean enhancement of SGC survival of $25.7 \%$ in stimulated cochleae compared with the deafened untreated cochleae. Thus, ES is an effective means for the protection of the SGC population after deafness, even if initiation of treatment is further delayed.

\section{SGC Survival in the GDNF/ES Group}

The main goal of this study was to determine the effects of a delayed and combined treatment with GDNF and ES on SGC survival after deafness. The results show that a combination of GDNF and ES is most effective and leads to a highly significant enhance-

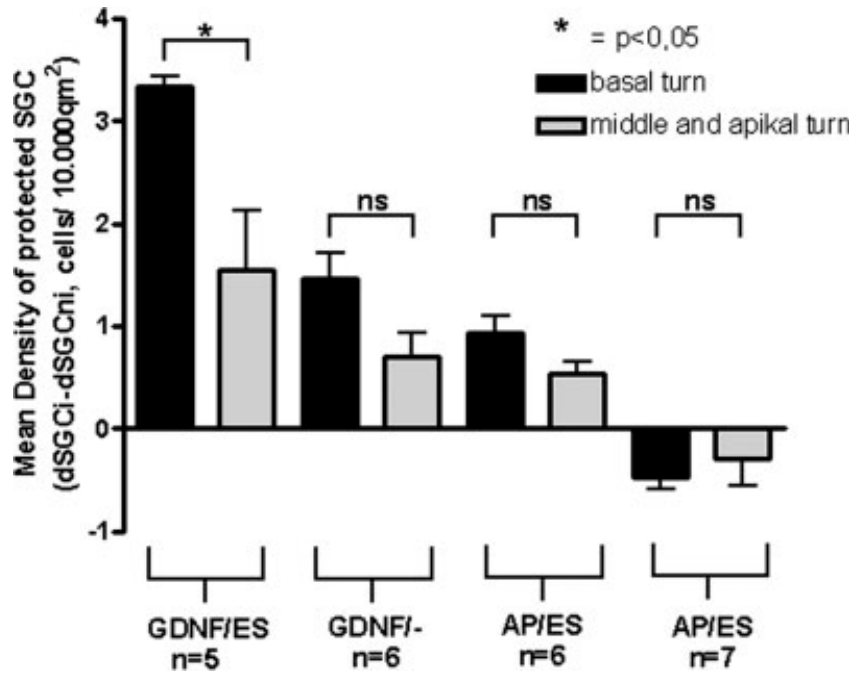

Fig. 6. An apparent difference in the survival of SGC is observed between the basal (lower and upper basal turn + first middle turn) and the middle and apical turn (second + third + fourth middle turn + apical turn) in all treatment groups. In the GDNF/ES, GDNF/ - and AP/ES group the mean density of protected SGC in the basal turn is higher than in the middle and apical turn. This disparity is statistically significant in the GDNF/ES cohort $(P<0.05)$. By contrast, the control cochleae (AP treated) showed a higher SGC density in the middle and apical turn when compared with the basal turn.

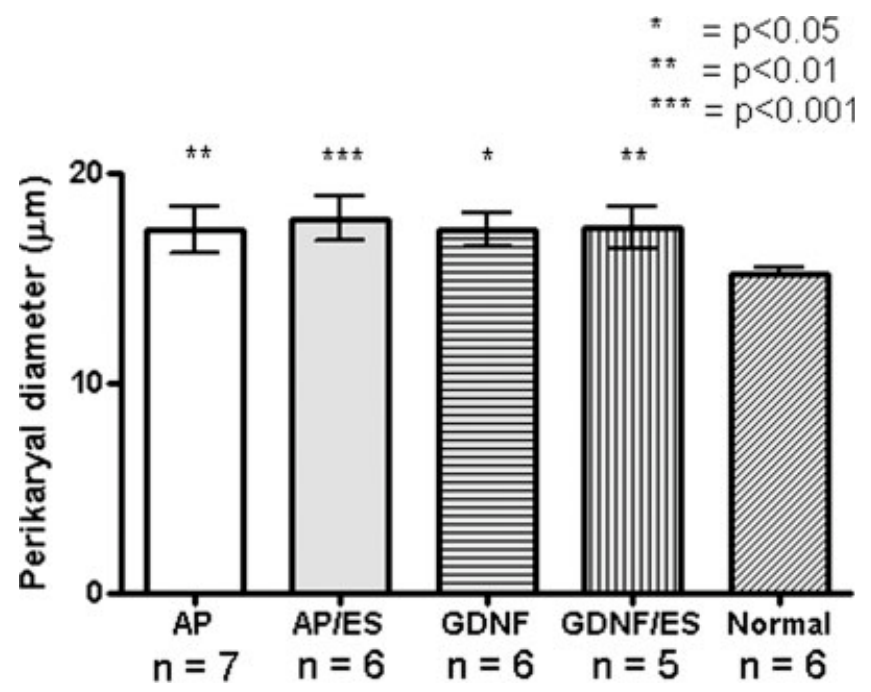

Fig. 7. Mean and SD of SGC perikaryal diameter for each treatment group and for normal hearing animals. No differences in cell diameter between the experimental groups were observed. Statistically significant differences between the normal hearing animals and the experimental groups are illustrated.

ment of SGC density even after a treatment delay of 24 days when compared with the control group. Furthermore, our data demonstrate that this delayed combined therapy protects SGC from degeneration after deafness 
significantly better than either intervention alone, and that this effect is more than additive.

Studies examining the effects of NTF in combination with chronic intracochlear ES on SGC survival have not included GDNF (Shepherd et al., 2005; Song et al., 2008). Only one previous study has investigated the effects of chronically combined GDNF and ES treatment (Kanzaki et al., 2002). By means of viral vectormediated gene delivery, they demonstrated that the combined treatment with GDNF and ES protects SGC from degeneration after hair cell loss. In that study, continuous ES was provided for 36 days, starting on day 8 after deafening with $100 \mu \mathrm{A}$ peak, $100 \mu \mathrm{sec} /$ phase, 250 $\mathrm{Hz}$, and $40 \%$ duty cycle. Their results showed that GDNF/ES treatment leads to a statistically significant increase of the mean density of SGC (nearly 11 cells/ $10,000 \mu^{2}$ ) when compared with treatment with GDNF or ES alone $(P<0.05)$. Our findings demonstrate a similar significant difference between the combined treatment with GDNF/ES and GDNF alone $(P<$ 0.05 , Fig. 6). However, we found a highly significant difference when comparing the GDNF/ES and AP/ES groups $(P<0.01)$. The stimulating parameters are nearly identical in both studies. Therefore, we suggest that the differences in SGC protection between the ES group and the GDNF/ES group are due to the different GDNF application methods. Kanzaki and colleagues (2002) tested the efficacy of GDNF transgene delivery mediated by an adenoviral vector. Adenoviral vectors were used to deliver NTFs to the cochlea; the concentration of the expressed protein can vary. Additionally, adenoviral transfection is known to be unstable ( $\mathrm{Lu}$ et al., 2005), and a significant decrease of protein expression may be expected over time. With the pump system for delivery of the pure protein, we were able to apply a defined GDNF concentration of $100 \mathrm{ng} / \mathrm{ml}$ throughout the whole study period.

\section{SGC Survival in the Control Group}

Application of AP to deafened cochleae had a negative effect on SGC survival. SGC density decreased in the left cochleae that were treated with AP by approximately $16 \%$ when compared with the deafened untreated right cochleae. This indicates that the survival of SGC might be affected by the implantation, the delivery procedure, or the AP itself. This finding is aided by Shepherd et al. (2005), who found lower SGC densities in deafened AP-treated control cochleae when compared with the contralateral untreated deafened ears of the same animal. Another possible explanation could be that NTFs, such as GDNF and artemin, are up-regulated in deafened cochleae (Wissel et al., 2006), but might be diluted by the delivery of AP. This effect could lead to an earlier onset of degeneration.

\section{Functional Evaluation}

The effects of chronic simultaneous application of NTFs and ES on SGC survival and neural threshold sen- sitivity in deafened guinea pigs have been examined in two previous studies.

Kanzaki et al. (2002) reported a slight reduction in threshold over time in all treatment groups, declaring that ES alone improves the functional state of existent SGC. In contrast, Shepherd et al. (2005) measured significant reduction of EABR thresholds only when the animals were treated with BDNF, either alone or in combination with ES. Our data corroborate the findings of Shepherd et al. that the combination of NTF intervention and ES reduces the EABR threshold over the treatment period. In contrast to their findings, the AP/ ES-treated animals of our study showed a threshold increase only until day 34; thereafter, the thresholds started to decrease. This suggests that the functional effect of the NTF starts immediately after factor application, while ES alone may have a delayed effect on EABR thresholds. The changes in the functional response are reflected in significant differences in the survival of SGC. Both electrically stimulated groups showed an increase in the density of SGC compared with the control group. However, the two GDNFtreated groups (GDNF/ES and GDNF/-) exhibited the greatest effects. These findings indicate again that GDNF is a more effective survival factor than ES for the protection of SGC and the maintenance of electrical excitability after deafness.

\section{Perikaryal Diameter}

Additional to our data, several previous reports demonstrated that exogenous NTF infusion into the deafened cochlea results in substantial increases in SGC soma size compared with normal hearing cochleae. For example, SGC soma were similar to or greater than those of normal hearing controls after BDNF infusion in guinea pigs (McGuinness and Shepherd, 2005; Shepherd et al., 2005) and significantly larger after infusion of both BDNF and acidic fibroblast growth factor together (Glueckert et al., 2008). Similar increase in neuronal size with GDNF treatment has also been reported, both in vivo (Zhou and $\mathrm{Wu}, 2006$ ) and in vitro (Anand et al., 2006). However, other authors showed no variation in size of the evaluated cells after GDNF treatment as well (Ducray et al., 2006). Furthermore, Dodson and Mohuiddin (2000) reported an initial increase of soma diameter within the first few weeks after deafness in the untreated groups. The mechanisms underlying an increased soma area after exogenous neurotrophin delivery or deafening remain unclear. Thus, the soma diameter after deafness and/or treatment is controversially discussed in the literature. Further studies are necessary to elucidate these morphological and molecular biological changes in SGC. Therefore, we only used the assessment of SGC diameter to rule out a possible effect of differences in cell size among the experimental groups in the determination of SGC density. 


\section{Conclusions}

Our results indicate that treatment with GDNF (100 $\mathrm{ng} / \mathrm{ml}$ ) as well as ES (intensity: $8 \mathrm{~dB}$ above EABR threshold) delayed by 3 weeks can significantly enhance survival and neurophysiological responsiveness of SGC after ototoxic drug treatment. The novel finding of this study is the highly synergistic trophic and functional effect of the delayed treatment with combined chronic ES and GDNF. Further experiments will be necessary to determine the most effective combination of GDNF concentrations and ES parameters. These investigations may have significant implications for a potential clinical application of a combined treatment with GDNF and ES via a cochlear implant in patients with severe sensorineural hearing loss.

\section{ACKNOWLEDGMENTS}

We thank MedEl GmbH for providing the used cochlear implant electrodes and Amgen Inc. for providing the GDNF. We also thank Chris Ellinger for designing and manufacturing the wearable chronic stimulators, and Peter Erfurt and Cornelia Roesl for their technical assistance.

\section{REFERENCES}

Anand U, Otto WR, Casula MA, Day NC, Davis JB, Bountra C, Birch R, Anand P. 2006. The effect of neurotrophic factors on morphology, TRPV1 expression and capsaicin responses of cultured human DRG sensory neurons. Neurosci Lett 399:51-56.

Araki S, Kawano A, Seldon L, Shepherd RK, Funasaka S, Clark GM. 1998. Effects of chronic electrical stimulation on spiral ganglion neuron survival and size in deafened kittens. Laryngoscope 108:687-695.

Dodson HC, Mohuiddin A. 2000. Response of spiral ganglion neurones to cochlear hair cell destruction in the guinea pig. J Neurocytol 29:525-537.

Ducray A, Krebs SH, Schaller B, Seiler RW, Meyer M, Widmer HR. 2006. GDNF family ligands display distinct action profiles on cultured GABAergic and serotonergic neurons of rat ventral mesencephalon. Brain Res 1069:104-112.

Ernfors P, Duan ML, ElShamy WM, Canlon B. 1996. Protection of auditory neurons from aminoglycoside toxicity by neurotrophin-3. Nat Med 2:463-467.

Gantz BJ, Woodworth GG, Knutson JF, Abbas PJ, Tyler RS. 1993. Multivariate predictors of success with cochlear implants. Adv Otorhinolaryngol 48:153-167.

Glueckert R, Bitsche M, Miller JM, Zhu Y, Prieskorn DM, Altschuler RA, Schrott-Fischer A. 2008. Deafferentation-associated changes in afferent and efferent processes in the guinea pig cochlea and afferent regeneration with chronic intrascalar brain-derived neurotrophic factor and acidic fibroblast growth factor. J Comp Neurol 507:1602-1621.

Hartshorn DO, Miller JM, Altschuler RA. 1991. Protective effect of electrical stimulation in the deafened guinea pig cochlea. Otolaryngol Head Neck Surg 104:311-319.

Jyung RW, Miller JM, Cannon SC. 1989. Evaluation of eighth nerve integrity by the electrically evoked middle latency response. Otolaryngol Head Neck Surg 101:670-682.

Kanzaki S, Stover T, Kawamoto K, Prieskorn DM, Altschuler RA, Miller JM, Raphael Y. 2002. Glial cell line-derived neurotrophic factor and chronic electrical stimulation prevent VIII cranial nerve degeneration following denervation. J Comp Neurol 454:350-360.
Kuang R, Hever G, Zajic G, Yan Q, Collins F, Louis JC, Keithley E, Magal E. 1999. Glial cell line-derived neurotrophic factor. Potential for otoprotection. Ann N Y Acad Sci 884:270-291.

Leake PA, Hradek GT, Rebscher SJ, Snyder RL. 1991. Chronic intracochlear electrical stimulation induces selective survival of spiral ganglion neurons in neonatally deafened cats. Hear Res 54:251-271.

Leake PA, Hradek GT, Snyder RL. 1999. Chronic electrical stimulation by a cochlear implant promotes survival of spiral ganglion neurons after neonatal deafness. J Comp Neurol 412:543-562.

Li L, Parkins CW, Webster DB. 1999. Does electrical stimulation of deaf cochleae prevent spiral ganglion degeneration? Hear Res 133:27-39.

Lousteau RJ. 1987. Increased spiral ganglion cell survival in electrically stimulated, deafened guinea pig cochleae. Laryngoscope 97(7 Pt 1):836842.

Lu FZ, Kitazawa Y, Hara Y, Jiang JY, Li XK. 2005. Long-term gene expression using the lentiviral vector in rat chondrocytes. Clin Orthop Relat Res 439:243-252.

Maruyama J, Yamagata T, Ulfendahl M, Bredberg G, Altschuler RA, Miller JM. 2007. Effects of antioxidants on auditory nerve function and survival in deafened guinea pigs. Neurobiol Dis 25:309-318.

McGuinness SL, Shepherd RK. 2005. Exogenous BDNF rescues rat spiral ganglion neurons in vivo. Otol Neurotol 26:1064-1072.

Miller JM, Altschuler RA. 1995. Effectiveness of different electrical stimulation conditions in preservation of spiral ganglion cells following deafness. Ann Otol Rhinol Laryngol Suppl 166:57-60.

Miller JM, Chi DH, O'Keeffe LJ, Kruszka P, Raphael Y, Altschuler RA. 1997. Neurotrophins can enhance spiral ganglion cell survival after inner hair cell loss. Int J Dev Neurosci 15:631-643.

Miller JM, Le Prell CG, Prieskorn DM, Wys NL, Altschuler RA. 2007. Delayed neurotrophin treatment following deafness rescues spiral ganglion cells from death and promotes regrowth of auditory nerve peripheral processes: effects of brain-derived neurotrophic factor and fibroblast growth factor. J Neurosci Res 85:1959-1969.

Mitchell A, Miller JM, Finger PA, Heller JW, Raphael Y, Altschuler RA. 1997. Effects of chronic high-rate electrical stimulation on the cochlea and eighth nerve in the deafened guinea pig. Hear Res 105:30-43.

Nadol JB Jr, Hsu WC. 1991. Histopathologic correlation of spiral ganglion cell count and new bone formation in the cochlea following meningogenic labyrinthitis and deafness. Ann Otol Rhinol Laryngol 100(9 Pt 1):712-716.

Nakaizumi T, Kawamoto K, Minoda R, Raphael Y. 2004. Adenovirusmediated expression of brain-derived neurotrophic factor protects spiral ganglion neurons from ototoxic damage. Audiol Neurootol 9:135-143.

Otte J, Schunknecht HF, Kerr AG. 1978. Ganglion cell populations in normal and pathological human cochleae. Implications for cochlear implantation. Laryngoscope 88(8 Pt 1):1231-1246.

Shepherd RK, Matsushima J, Martin RL, Clark GM. 1994. Cochlear pathology following chronic electrical stimulation of the auditory nerve: II. Deafened kittens. Hear Res 81:150-166.

Shepherd RK, Coco A, Epp SB, Crook JM. 2005. Chronic depolarization enhances the trophic effects of brain-derived neurotrophic factor in rescuing auditory neurons following a sensorineural hearing loss. J Comp Neurol 486:145-158.

Shinohara T, Bredberg G, Ulfendahl M, Pyykko I, Olivius NP, Kaksonen R, Lindstrom B, Altschuler R, Miller JM. 2002. Neurotrophic factor intervention restores auditory function in deafened animals. Proc Natl Acad Sci U S A 99:1657-1660.

Song BN, Li YX, Han DM. 2008. Delayed electrical stimulation and BDNF application following induced deafness in rats. Acta Otolaryngol:1-13.

Spoendlin H. 1984. Factors inducing retrograde degeneration of the cochlear nerve. Ann Otol Rhinol Laryngol Suppl 112:76-82. 
Staecker H, Kopke R, Malgrange B, Lefebvre P, Van de Water TR. 1996. NT-3 and/or BDNF therapy prevents loss of auditory neurons following loss of hair cells. Neuroreport 7:889-894.

Stover T, Yagi M, Raphael Y. 1999. Cochlear gene transfer: round window versus cochleostomy inoculation. Hear Res 136:124-130.

Tyler RS, Summerfield AQ. 1996. Cochlear implantation: relationships with research on auditory deprivation and acclimatization. Ear Hear 17(3 Suppl):38S-50S.

Webster M, Webster DB. 1981. Spiral ganglion neuron loss following organ of Corti loss: a quantitative study. Brain Res 212:17-30.

West BA, Brummett RE, Himes DL. 1973. Interaction of kanamycin and ethacrynic acid. Severe cochlear damage in guinea pigs. Arch Otolaryngol 98:32-37.

Wissel K, Wefstaedt P, Rieger H, Miller JM, Lenarz T, Stover T. 2006. Upregulation of glial cell line-derived neurotrophic factor and artemin
mRNA in the auditory nerve of deafened rats. Neuroreport 17:875878.

Yagi M, Kanzaki S, Kawamoto K, Shin B, Shah PP, Magal E, Sheng J, Raphael Y. 2000. Spiral ganglion neurons are protected from degeneration by GDNF gene therapy. J Assoc Res Otolaryngol 1:315-325.

Yamagata T, Miller JM, Ulfendahl M, Olivius NP, Altschuler RA, Pyykko I, Bredberg G. 2004. Delayed neurotrophic treatment preserves nerve survival and electrophysiological responsiveness in neomycindeafened guinea pigs. J Neurosci Res 78:75-86.

Ylikoski J, Pirvola U, Virkkala J, Suvanto P, Liang XQ, Magal E, Altschuler R, Miller JM, Saarma M. 1998. Guinea pig auditory neurons are protected by glial cell line-derived growth factor from degeneration after noise trauma. Hear Res 124:17-26.

Zhou LH, Wu W. 2006. Survival of injured spinal motoneurons in adult rat upon treatment with glial cell line-derived neurotrophic factor at 2 weeks but not at 4 weeks after root avulsion. J Neurotrauma 23:920-927. 